\title{
Detecção de dois tipos tumorais distintos em cavidade bucal de Cão: Relato de caso
}

\author{
Detection of two different tumor types in dog oral cavity: Case report \\ Detección de dos tipos distintos de tumores en la cavidad bucal de un perro: Reporte de caso
}

Recebido: 08/05/2021 | Revisado: 16/05/2021 | Aceito: 19/05/2021 | Publicado: 06/06/2021

\author{
Geovanna Santos Pereira \\ ORCID: https://orcid.org/0000-0001-5981-3709 \\ Centro Universitário de Mineiros, Brasil \\ E-mail: geovannasantos846@gmail.com \\ Monique Resende Carvalho \\ ORCID: https://orcid.org/0000-0001-8217-938X \\ Centro Universitário de Mineiros, Brasil \\ E-mail: monique_r_c@ @otmail.com \\ Samira Lessa Abdalla \\ ORCID: https://orcid.org/0000-0001-5657-3631 \\ Hospital Cães e Gatos 24 horas, Brasil \\ E-mail: abdallasamira@hotmail.com \\ Marta Fernanda Albuquerque da Silva \\ ORCID: https://orcid.org/0000-0002-5651-3855 \\ Universidade Federal Rural do Rio de Janeiro, Brasil \\ E-mail:mfas@unisys.com.br \\ Lianna Ghisi Gomes \\ ORCID: https://orcid.org/0000-0003-0909-2426 \\ Universidade Federal de Mato Grosso, Brasil \\ E-mail: liannaghisi@gmail.com \\ Paulo Roberto Spiller \\ ORCID: https://orcid.org/0000-0002-4659-4647 \\ Universidade de Cuiabá, Brasil \\ E-mail: paulo_spiller@hotmail.com \\ Eduardo Ferreira Faria \\ ORCID: https://orcid.org/0000-0003-1108-7933 \\ Universidade de Cuiabá, Brasil \\ E-mail: eduffaria@gmail.com \\ Priscila Chediek Dall'Acqua \\ ORCID: https://orcid.org/0000-0001-5781-4492 \\ Centro Universitário de Mineiros, Brasil \\ E-mail: priscila.chediek@unifimes.edu.br \\ Eric Mateus Nascimento de Paula \\ ORCID: https://orcid.org/0000-0002-5948-1860 \\ Centro Universitário de Mineiros, Brasil \\ E-mail: ericmateus@unifimes.edu.br \\ Andresa de Cássia Martini \\ ORCID: https://orcid.org/0000-0002-2553-3685 \\ Centro Universitário de Mineiros, Brasil \\ E-mail: andresa.martini@unifimes.edu.br
}

\begin{abstract}
Resumo
A cavidade bucal representa o quarto local mais frequente de neoplasias, sendo responsáveis por aproximadamente $5 \%$ dos tumores em cães. Sua ocorrência é maior em animais com idade média a avançada, sendo também observada em animais jovens. O objetivo desse relato foi realizar a descrição de dois tipos tumorais distintos identificados na cavidade bucal de um cão, dando ênfase para realização de exame histopatológico individual, para diferentes massas em uma mesma região anatômica. Foi avaliado um cão da raça Golden Retriever e ao exame específico da cavidade bucal foi observado halitose, doença periodontal grau III e presença de neoformação em mucosa jugal da maxila e mucosa mandibular, ambas do lado esquerdo. $\mathrm{O}$ animal foi encaminhado para realização de exérese de massa, através de técnica cirúrgica clássica e os fragmentos encaminhados para análise histopatológica, que evidenciou melanoma melanocítico em mucosa mandibular e mastocitoma na mucosa jugal da maxila, ambas do lado esquerdo. O pósoperatório consistiu na limpeza da cavidade bucal duas vezes ao dia com periogard ${ }^{\circ}$, durante dez dias, stomorgyl 20®, 3 comprimidos, SID, por 7 dias, maxicam® $2 \mathrm{mg}, 2$ comprimidos, SID, durante 3 dias e tramadol $50 \mathrm{mg}, 2$ comprimidos, BID, por 5 dias, todos por via oral. Ao $10^{\circ}$ dia do pós-operatório o animal retornou para avaliação, onde observou-se boa cicatrização no local de exérese das lesões, nessa ocasião foi realizada uma sessão de eletroquimioterapia com sulfato de bleomicina, na região da cicatriz cirúrgica. Aos 30 dias do pós- operatório o animal encontrava-se bem, com boa alimentação e sem recidiva de lesões em cavidade bucal. Esse estudo reforça a
\end{abstract}


necessidade do diagnóstico e adoção terapêutica rápidas, para manutenção da qualidade e aumento na expectativa de vida do paciente que apresenta neoplasia em cavidade bucal. A realização de exame histopatológico individual, em diferentes massas em uma mesma região anatômica é imprescindível, a fím de identificar o tipo tumoral corretamente e estabelecer o tratamento, que pode contemplar exérese cirúrgica clássica ou ainda utilizar de terapias adjuvantes, como a eletroquimioterapia.

Palavras-chave: Mandíbula; Neoplasia; Oncologia veterinária.

\begin{abstract}
The oral cavity represents the fourth most frequent site of neoplasms, being responsible for approximately $5 \%$ of tumors in dogs. Its occurrence is higher in animals with medium to advanced age, being also observed in young animals. The purpose of this report was to describe two distinct tumor types identified in a dog's oral cavity, with emphasis on individual histopathological examination, for different masses in the same anatomical region. A Golden Retriever dog was evaluated and a specific examination of the oral cavity showed halitosis, periodontal disease grade III and the presence of new formation in the maxillary cheek mucosa and mandibular mucosa, both on the left side. The animal was sent for mass excision, using the classic surgical technique and the fragments were sent for histopathological analysis, which showed melanocytic melanoma in the mandibular mucosa and mastocytoma in the maxillary mucosa of the maxilla, both on the left side. The postoperative period consisted of cleaning the oral cavity twice daily with periogard $\AA$, for ten days, stomorgyl 20®, 3 tablets, SID, for 7 days, maxicam ${ }^{\circledR} 2$ mg, 2 tablets, SID, for 3 days and tramadol $50 \mathrm{mg}, 2$ tablets, BID, for 5 days, all orally. On the 10th postoperative day, the animal returned for evaluation, where good healing was observed at the lesion excision site, on that occasion an electrochemotherapy session with bleomycin sulfate was performed, in the region of the surgical scar. At 30 days after the operation, the animal was well, with good food and no recurrence of lesions in the oral cavity. This study reinforces the need for rapid diagnosis and therapeutic adoption, in order to maintain quality and increase the life expectancy of patients who present with neoplasia in the oral cavity. The performance of an individual histopathological examination, in different masses in the same anatomical region, is essential in order to correctly identify the tumor type and establish the treatment, which may include classical surgical excision or use adjuvant therapies, such as electrochemotherapy.
\end{abstract}

Keywords: Jaw; Neoplasia; Veterinary oncology.

\title{
Resumen
}

La cavidad oral representa el cuarto sitio más frecuente de neoplasias, siendo responsable de aproximadamente el $5 \%$ de los tumores en perros. Su ocurrencia es mayor en animales de mediana a avanzada edad, observándose también en animales jóvenes. El propósito de este informe fue describir dos tipos de tumores distintos identificados en la cavidad oral de un perro, con énfasis en el examen histopatológico individual, para diferentes masas en la misma región anatómica. Se evaluó a un perro Golden Retriever y un examen específico de la cavidad oral mostró halitosis, enfermedad periodontal grado III y la presencia de nueva formación en la mucosa de la mejilla maxilar y mucosa mandibular, ambas del lado izquierdo. El animal fue referido para exéresis masiva, mediante la técnica quirúrgica clásica y los fragmentos fueron enviados para análisis histopatológico, que mostró melanoma melanocítico en mucosa mandibular y mastocitoma en mucosa maxilar del maxilar, ambos del lado izquierdo. El postoperatorio consistió en la limpieza de la cavidad bucal dos veces al día con periogard®, durante diez días, stomorgyl $20 ®, 3$ comprimidos, SID, durante 7 días, maxicam ${ }^{\circledR} 2 \mathrm{mg}, 2$ comprimidos, SID, durante 3 días y tramadol $50 \mathrm{mg}$, 2 comprimidos, dos veces al día, durante 5 días, todos por vía oral. Al décimo día postoperatorio el animal regresó para evaluación, donde se observó buena cicatrización en el sitio de escisión de la lesión, en esa ocasión se realizó una sesión de electroquimioterapia con sulfato de bleomicina, en la región de la cicatriz quirúrgica. A los 30 días de la operación, el animal se encontraba bien, con buena alimentación y sin recurrencia de lesiones en la cavidad bucal. Este estudio refuerza la necesidad de un diagnóstico rápido y de adopción terapéutica, con el fin de mantener la calidad y aumentar la esperanza de vida de los pacientes que presentan neoplasia en la cavidad oral. La realización de un examen histopatológico individual, en diferentes masas de una misma región anatómica, es fundamental para identificar correctamente el tipo de tumor y establecer el tratamiento, que puede incluir la exéresis quirúrgica clásica o el uso de terapias adyuvantes, como la electroquimioterapia.

Palabras clave: Mandíbula; Neoplasia; Oncología veterinária.

\section{Introdução}

A cavidade bucal é bastante acometida por inúmeras patologias, representando o quarto local mais frequente de neoplasias, que são responsáveis por aproximadamente 5\% dos tumores em cães (Fossum, 2008). A prevalência de tumores de cavidade bucal em cães é maior em machos e normalmente com idade média a avançada, sendo o melanoma o tumor maligno de maior frequência, seguido de mastocitoma, carcinoma de células escamosas e fibrossarcoma (Dias et al., 2013).

O melanoma é um dos tumores malignos da cavidade bucal mais frequente em cães, representando cerca de 30-40\% 
de todas as afecções deste tipo (Requicha et al., 2015). É definido como uma desordem neoplásica proveniente de uma transformação maligna do melanócito, responsável pela produção de melanina, um pigmento de coloração marrom escura. Este pigmento é mantido dentro de uma estrutura chamada melanossoma, que se acumula dentro do citoplasma chamada de queratinócitos (Gineste, 2016; Barreto et al., 2017).

De acordo com Lindoso et al. (2017) os melanócitos se multiplicam de forma autônoma, fugindo do controle dos queratinócitos, apresentando crescimento difuso e descontrolado, formando assim os tumores sólidos que podem ser apresentados como benignos e malignos. O melanoma maligno envolve várias fases, iniciando de forma benigna, pelas manchas na pele até a formação de um tumor invasivo e metastático (Manzan et al., 2005). Pode ocorrer metástase por duas vias, sendo elas via linfática, para os linfonodos e hematológicos para os pulmões (Freitas et al., 2007).

O melanoma tem predileção em raças fortemente pigmentadas, como as raças Terrier Escocês, Boxer, Golden Retriever, Schnauzer miniatura, Doberman, Pinscher, Chihuahua e Chow Chow (Lindoso et al., 2017).

Seguido do melanoma, o mastocitoma também é um dos tumores malignos mais frequente que acometem os cães, representando de 11-27\% dos casos (Furlani et al., 2008). De acordo com Natividade et al. (2014) seu comportamento biológico é variado, podendo apresentar desde única nodulação com comportamento benigno, até com comportamento metastático extremamente maligno.

O mastocitoma é uma neoplasia de células redondas, caracterizada pela proliferação anormal de mastócitos, que são encontrados principalmente nos tecidos subcutâneos e mucosas e podendo ser local ou sistêmica. (Daleck, 2017; Machado, 2018). Podem acometer algumas raças, principalmente as braquicefálicas, porém algumas raças apresentam maior incidência como Boxer, Bulldog, Labrador, Teckel, Sharpei e Golden Retriever (Prado, 2012; Machado, 2018). Segundo Prado et al. (2012) os mastocitomas não possuem predileção sexual, porém tem frequências em cães com idade avançada, não descartando ocorrência em animais jovens.

Os sinais clínicos dos animais que apresentam neoplasias bucais podem incluir disfagia, dentes frouxos, deformação facial, além disso podem apresentar também halitose, sialorreia, perda de apetite e perda de peso. (Gineste, 2016). De acordo com Gomes et al. (2009) para o diagnóstico definitivo destas neoplasias é necessário a avaliação histopatológica e o tratamento pode ser feito através de quimioterapia, radioterapia e cirurgia, sendo a cirurgia o principal procedimento terapêutico e diagnóstico dos tumores bucais.

Diante de uma evidência neoplásica é importante ressaltar o uso da eletroquimioterapia como um dos tratamentos mais utilizados, seguido da radioterapia e a cirurgia, sendo como principal procedimento (Gomes et al., 2009).

A eletroquimioterapia nada mais é do que um protocolo terapêutico que tem como opção tratar neoplasias (Paula, 2019). Usa-se uma combinação de duas técnicas: "quimioterapia e a aplicação de campos elétricos" para poder potencializar a ação dos fármacos assim reduzindo a regressão de tumores (Cruz, 2018). De acordo com Zeferino (2019) a eletroquimioterapia consente a administração dos agentes quimioterápicos por via IV (intravenosa). O uso da eletroquimioterapia em animais está sendo uma opção alternativa no tratamento de neoplasias e mostrando-se totalmente eficaz (Zeferino, 2019).

Diante do exposto, o objetivo desse relato é a descrição da identificação de dois tipos tumorais distintos na cavidade bucal de um cão da raça Golden Retriever, dando ênfase para realização de exame histopatológico individual, em diferentes massas em uma mesma região anatômica.

\section{Metodologia}

Foi atendido em um serviço veterinário privado um cão da raça Golden Retriever, 13 anos, sendo a queixa da tutora o mau cheiro na boca, queda de apetite e aumento de volume da região. Na avaliação clínica geral não foram identificadas alterações, sendo que ao exame físico os parâmetros fisiológicos, hemograma e bioquímica sérica, estavam dentro da 
normalidade. Ao exame específico da cavidade bucal observou-se halitose, doença periodontal grau III segundo AVDC (American Veterinary Dental Collegee. https://www.avdc.org/Nomenclature/Nomen-Intro.html) e presença de neoformação em mucosa jugal da maxila e mucosa mandibular, ambas do lado esquerdo.

O animal foi encaminhado para realização de exérese de massa, através de técnica cirúrgica clássica (Fossum, 2014), sendo que o fragmento maxilar apresentava- se com consistência firme, forma irregular, coloração acastanhada, medindo cerca de 3,2 x 1,0 x $2,0 \mathrm{~cm}$ de comprimento. $\mathrm{O}$ fragmento mandibular apresentou consistência firme, forma irregular multilobular, superfície externa de coloração enegrecida, medindo 3,0 x 1,5 x 3,5 de comprimento, ambas foram encaminhadas para exame histopatológico em serviço especializado.

O exame evidenciou para o fragmento maxilar (Fig. 1A), epitélio de revestimento mucoso com hiperplasia irregular, no córion proliferação neoplásica com mastócitos distribuídos de maneira predominantemente difusa ou formando agrupamentos localizados no córion superficial, córion profundo e porções baixas do fragmento. As células neoplásicas apresentaram núcleos arredondados de tamanho moderado e com nucléolos inconspícuos. Os citoplasmas revelaram-se amplos com grânulos basofílicos densos. A relação núcleo citoplasma apresentava-se preservada na maioria das células. A contagem de figuras de mitose em 10 campos de $400 \mathrm{X}$ foi igual a zero. Foi observado infiltrado inflamatório eosinofílico moderado à severo entremeado aos mastócitos neoplásicos, sendo as margens comprometidas por células neoplásicas, com quadro morfológico compatível com mastocitoma.

Para o fragmento mandibular (Figura 1B) foi evidenciado lesão sobrelevada revestida por epitélio de revestimento mucoso íntegro com presença de ninhos de melanócitos atípicos em camadas baixas e altas do mesmo. No córion observado proliferação neoplásica moderadamente circunscrita constituída por melanócitos, apresentando disposição em pequenos feixes celulares curtos com arranjo randômico e agrupamentos. As células neoplásicas revelam citoplasmas com bordos mal definidos, alongados, poligonais e contendo variável quantidade de grânulos melanocíticos. Os núcleos foram arredondados a ovalados, com nucléolos ora inconspícuos, ora evidentes e proeminentes. Figuras de mitoses foram observadas em alguns campos presença de 9 figuras de mitoses em 10 campos de $400 \mathrm{X}$ em áreas mais mitoticamente ativas em melanócitos fusiformes, as margens cirúrgicas livres de células neoplásicas, com quadro morfológico compatível com tumor melanocítico oral, favorecendo melanoma melânico.

Figura 1A- Fragmento de consistência firme, forma irregular, coloração acastanhada em região maxilar esquerda. 1BFragmento mandibular de consistência firme, forma irregular multilobular, superfície externa de coloração enegrecida.

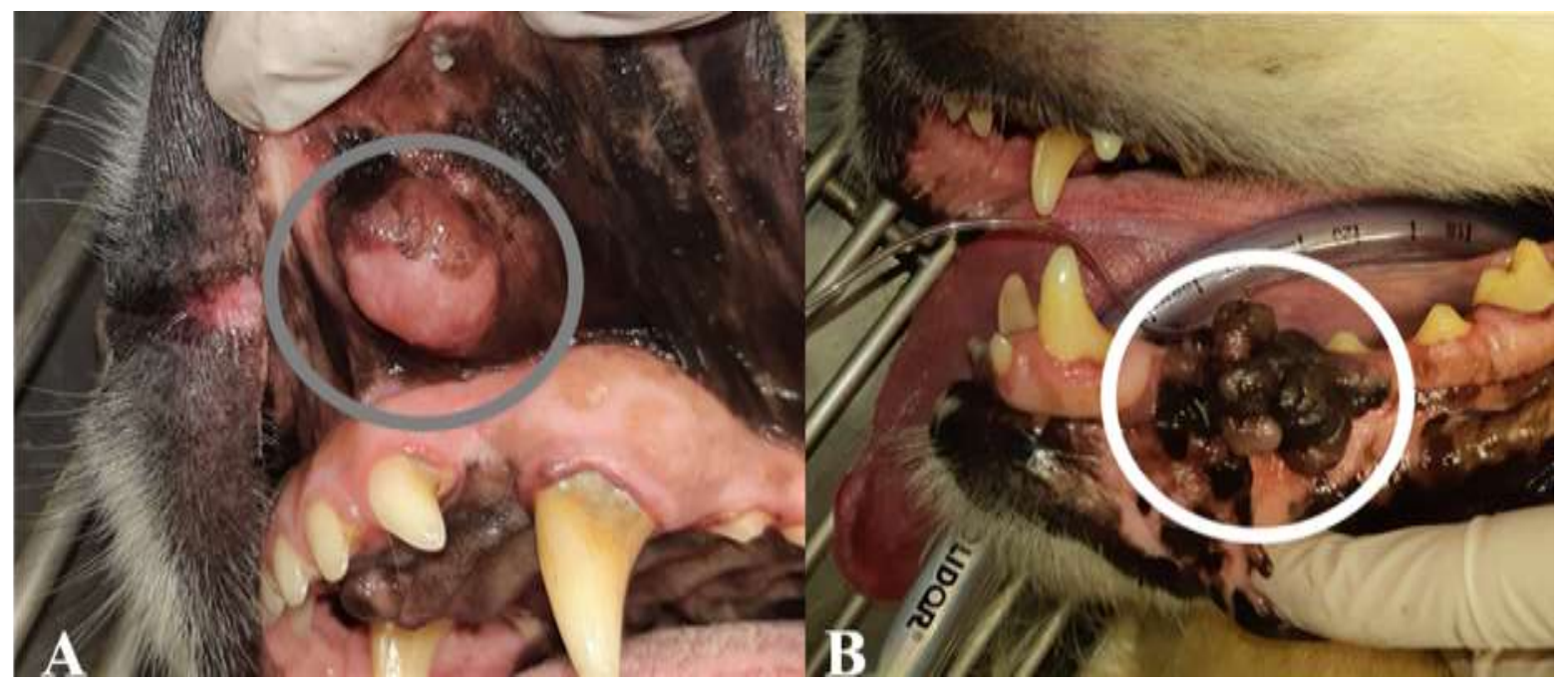

Fonte: Autores. 
O pós-operatório consistiu na limpeza da cavidade bucal duas vezes ao dia com periogard®, durante dez dias, stomorgyl 20®, 3 comprimidos, SID, por 7 dias, maxicam® $2 \mathrm{mg}, 2$ comprimidos, SID, durante 3 dias e tramadol $50 \mathrm{mg}, 2$ comprimidos, BID, por 5 dias, todos por via oral.

Ao $10^{\circ}$ dia do pós operatório o animal retornou para avaliação, onde observou-se boa cicatrização no local de exérese das lesões, nessa ocasião foi realizada uma sessão de eletroquimioterapia com sulfato de bleomicina, na região da cicatriz cirúrgica. Aos 30 dias do pós operatório o animal encontrava-se bem, com boa alimentação e sem recidiva de lesões em cavidade bucal. Orientado as reavaliações periódicas por um período de 6 meses.

\section{Resultados e Discussão}

As neoplasias que afetam a cavidade bucal dos animais não estão totalmente esclarecidas, pois acredita-se que essas estão divididas entre fatores ambientais e internos. Os ambientais envolvem a radiação, carcinógenos químicos como, herbicidas e inseticidas, já os fatores internos envolvem as infecções, fatores hormonais e genéticos (Dias, 2013). O animal em questão não apresentou histórico de causas ambientais que pudessem ter levado as neoplasias, e fatores internos não puderam ser adequadamente identificados, uma vez que o animal já foi levado ao serviço veterinário apresentando lesões avançadas, com necessidade de rápida intervenção cirúrgica. Assim torna-se evidente a importância de uma avaliação diagnóstica para os tumores da cavidade bucal devido a ampla variedade de tipos tumorais e comportamento biológico (Silva, 2016).

A idade média para ocorrência de neoplasias na cavidade oral é de 10 anos (Fernandes et al., 2015). De acordo com Chaves et al. (2020), tumores na cavidade bucal e região cervical em cães de idade avançada são comuns, assim como relatado nesse caso, onde o paciente apresentava idade avançada. Ainda a exemplo do paciente desse relato, Fossum (2014) afirma que os tumores malignos orais possuem um risco relativo maior de ocorrência em machos do que em fêmeas.

Esse estudo corrobora com Chaves et al. (2020) onde afirmam que para um diagnóstico definitivo a análise histológica do tecido tumoral deve ser realizada, a exemplo desse relato, onde através do exame histológico, foram identificados 2 tipos tumorais distintos em evolução simultânea. Gomes e colaboradores (2017), descreveram um caso de diferentes tipos de neoplasias benignas bucal em um cão idoso, portanto a que se dar ênfase para realização de exame histopatológico individual, em diferentes massas em uma mesma região anatômica, a fim de identificar corretamente o tipo tumoral e a terapia ideal.

Segundo Dias et al. (2013) as neoplasias são a maior causa de morte nos animais, sendo que a cavidade bucal representa ao quarto lugar mais afetado em comparação as outras regiões (glândula mamária, órgãos genitais e pele). Dentre essas neoplasias malignas citadas neste relato, melanoma e o mastocitoma, existem outros que são frequentes na cavidade bucal dos cães, como o fibrossarcoma e carcinoma de células escamosas (Luz, 2017). A maioria dessas neoplasias tem comportamento invasivo, sendo a excisão cirúrgica uma abordagem terapêutica mais eficaz e rápida pois como adotado neste caso o indicado é a remoção total do tumor se possível (Machado, 2019; Pedreira, 2019).

As exéreses de tumores de cavidade bucal tendem a ser extensas e dolorosas, neste caso o uso de analgésicos é essencial para que o animal tenha uma boa evolução e recuperação cirúrgicas. A utilização de analgésicos no pós operatório diminui a ansiedade, estresse devido a dor, possibilita conforto apropriado e um tempo de recuperação mais rápido (Souza, et al., 2018). A exemplo do utilizado nesse caso que adotou anti-inflamatório e analgésico, obtendo controle da dor satisfatório.

Para esses tipos tumorais além da excisão cirúrgica ser uma forma de tratamento, existem outras formas que são utilizadas, como neste caso que adotou o uso da eletroquimioterapia (EQT) coadjuvante. O uso da eletroquimioterapia na Medicina Veterinária vem se destacando como uma opção de tratamento de primeira linha, como sua simplicidade, rapidez de aplicação, baixa toxicidade e raros efeitos adversos associados (Zeferino, 2019). 
A utilização de bleomicina intralesional em melanomas de cavidade oral de cães, promoveu alta taxa de resposta, com 70\% de controle local, segundo estudos prévios (Spugnini et al., 2006), assim a adoção da EQT pode ter contribuído positivamente com o prognóstico do caso em questão, ademais Mali (2013) afirma em seu estudo que a eficácia promovida pela EQT é variável e remissões significativas podem ser observadas em diferentes tipos tumorais em homens e animais.

Gineste (2016), afirma que o mastocitoma e melanoma, geralmente apresentam comportamento invasivo, de crescimento rápido, agressivos, tornando um prognóstico reservado a desfavorável. Para esse caso a orientação de acompanhamento periódico durante 6 meses foi realizada, a fim de observar criteriosamente recidivas tumorais.

\section{Conclusão}

Esse relato reforça a necessidade do diagnóstico e adoção terapêutica rápidas, para manutenção da qualidade e aumento na expectativa de vida do paciente que apresenta tumor em cavidade bucal. A realização de exame histopatológico individual, em diferentes massas em uma mesma região anatômica é imprescindível, a fim de identificar o tipo tumoral corretamente e estabelecer o tratamento, que pode contemplar exérese cirúrgica clássica ou ainda utilizar de terapias adjuvantes, como a eletroquimioterapia.

\section{Referências}

American Veterinary Dental College. Veterinary Dental Nomenclature. https://www.avdc.org/Nomenclature/Nomen-Intro.html.

Barreto, H. M., Trintade, D. C., Mazzeo, R. A., Oliveira, J. M., Custodio, A. C. F., \& Sá, M. A. F. (Ed) (2017). Aspecto patológico, clínico, cirúrgico e terapêuticos do melanoma oral em cão: Relato de caso. Revista Científica de Medicina Veterinária ano XVI, n.28. Centro Universitário de Barra Mansa, Rio de Janeiro.

Cruz, L. M. (2018). Utilização da eletoquimioterapia no tratamento do mastocitoma canino (Trabalho de conclusão de curso). Universidade Federal do Rio Grande do Sul, Faculdade de Veterinária, Porto Alegre, Rio Grande do Sul, Brasil.

Chaves, L. D. C. S., Silva, F. L., Silva, C. R. A., Sousa, J. M. C., Oliveira, J. R. A., Silva, L. S., \& Santos, L. S. (Ed) (2020). Tratamento cirúrgico de neoplasias em cão na cavidade oral e região cervical: Relato de caso. Publicações em Medicina Veterinária e Zootecnia. 14(1), 1-6.

Dias, F. G. G., Dias, L. G. G. G., Pereira, L. F., Cabrini, T. M., \& Rocha, J. R. (Ed) (2013). Neoplasias orais nos animais de companhia - Revisão de liteatura. Revista Científica Eletrônica de Medicina Veterinária. ano XI, n.20. Faculdade de Medicina Veterinária e Zootecnia de Garça.

Fernandes, C. C., Medeiros, A. A., Magalhaes, G. M., Szabo, M. P. J., Queiroz, R. P., Silva, M. V. A., \& Soares, N. P. (Ed) (2015). Frequência de neoplasias cutâneas em cães atendidos no hospital veterinário da Universidade Federal de Uberlândia durante os anos 2000 a 2010. Bioscience Journal, (31(2):541-548).

Fossum, T. W. (2014). Cirurgia de pequenos animais. (4a ed). Elsevier.

Furlani, J. M., Daleck, C. R., Vicenti, F. A. M., Nerdi, A. B., Pereira, G. T., Santana, D., \& Silva, L. A. F. (Ed) (2008). Mastocitoma canino: Relato de caso. Ciência Animal Brasileira, 9(1), 242-250. UNESP.

Freitas, S. H., Doria, R. G. S., Pires, M. A. M., Mendonça, F. S., Camargo, L. M., \& Neto, J. E. (Ed) (2007). Melanoma oral maligno em cadela: Relato de caso. Veterinária em foco - Canoas, 5(1), 16-21. Faculdade de Medicina Veterinária de Universidade de Cuiabá-UNIC.

Gineste, D. L. (2016). Melanoma Orais em Cães: Relato de caso (Trabalho de Conclusão de Curso). Centro Universitário CESMAC, Curitiba, Paraná, Brasil.

Gomes, C., Oliveira, L. O., Elizeire, M. B., Oliveira, M. B., Oliveira, R. T., \& Contesini, E. A. (Ed) (2009). Avaliação epidemiológica de cães com neoplasias orais atendidos no hospital de clínica veterinária da Universidade Federal do Rio Grande do Sul. Ciência Animal Brasileira, 10(3), 835-839. Universidade Federal do Rio Grande do Sul.

Gomes, L. G., Martini, A. C., Menegassi, C. C., Abdalla, S. L., Moura, A. P. B. D., Souza, R. L., \& Silva, M. F. A. (2017). Diferentes neoplasias benignas oral em cão. In: $38^{\circ}$ Congresso Brasileiro da ANCLIVEPA, 2017, Recife. $38^{\circ}$ Congresso Brasileiro da ANCLIVEPA.

Lindoso, J. V. S., Rufino, A. K. B., Luz, P. M. S., Silva, T. S., Junior, F. L. S., Sousa, F. B., \& Sales, K. K. S. (Ed) (2017). Melanoma metastático em cão: Relato de caso. Publicações em Medicina Veterinária e Zootecnia, 11(1), 346-350. Universidade Federal do Piauí.

Luz, A. C. A. (2017). Neoplasias orais em cães diagnosticadas no laboratório de patologia animal do hospital veterinário da universidade federal de uberlandia: estudo retrospectivo (Trabalho de Conclusão de Curso). Universidade Federal de Uberlandia, Faculdade de Medicina Veterinária, Uberlândia, Minas Gerais, Brasil.

Machado, R. P. (2018). Ocorrência de mastocitoma em cães atendidos no hospital veterinário Phatos em Porto Alegre no ano de 2018 (Trabalho de Conclusão de Curso). Universidade do Sul de Santa Catarina, Porto Alegre, Rio Grande do Sul, Brasil. 
Research, Society and Development, v. 10, n. 6, e43710615905, 2021

(CC BY 4.0) | ISSN 2525-3409 | DOI: http://dx.doi.org/10.33448/rsd-v10i6.15905

Mali, B., Jarm, T., Snoj, M. et al. (Ed) (2013). Antitumor effectiveness of electrochemotherapy: a systematic review and meta-analysis. European Journal Surgery Oncology. (39: 4-16).

Manzan, R. M., Junior, A. R. S., Perinelli, S. C., Bertoncelli, M. F., \& Zica, V. P. (Ed) (2005). Considerações sobre melanoma maligno em cães: Uma abordagem histológica. Bol. Med. Vet, 1(1).

Natividade, F. S., Castro, M. B., Silva, A. S., Oliveira, L. B., Mcmanus, C. M., Galera, P. D. (Ed) (2014). Análise de sobrevida e fatores prodnósticos de cães com mastocitoma cutâneo. Pesquisa Veterinária Brasileira, (pp.874-884).

Paula, R. C. L. (2019). Eletroquimioterapia em cães - Revisão bibliográfica (Trabalho de Conclusão de Curso). Centro Universitário do Planalto Apparecido dos Santos - UNICEPLAC, Gama. Brasília, Distrito Federal, Brasil.

Pedreira, G. G. F. M. (2019). Identificação e análise de várias com influência no intervalo livre de doenças pós-cirúrgico em neoplasias malignas orais no cãoum estudo retrospectivo (Dissertação de Mestrado Integrado em Medicina Veterinária). Universidade de Lisboa, Faculdade de Medicina Veterinária, Lisboa, Portugal.

Prado, A. A. F., Leao, D. A., Ferreira, A. O., Machado, C., \& Maria, D. A. (Ed) (2012). Mastocitoma em cães: Aspecto clínicos, histopatológicos e tratamento. Enciclopédia Biosfera (n.14, Vol.8, pp.2151-2164). Faculdade de Medicina Veterinária e Zootecnia da Universidade de São Paulo. Centro Científico Conhecer - Goiânia.

Requicha, J. F., Pires, M. A., Albuquerque, C. M., \& Viegas, C. A. (Ed) (2015). Neoplasia da cavidade oral do cão - Breve revisão. Revista Brasileira Medicina Veterinária, (pp.41-46). Faculdade de Medicina Veterinária, Universidade de Humanidades e Tecnologias, Campo Grande.

Silva, L. G. (2016). Descrição clínico-cirúrgico de seis casos de maxilectomia parcial para o tratamento de neoplasias em cavidade oral em cães (Trabalho de Coclusão de Curso). Universidade Federal de Mato Grosso, Cuiabá, Brasil.

Souza, P. S., Miliozzi G., Rodrigues, C. A., Franco, M., \& Sabino, F. A. (Ed) (2018). Abordagem terapêutica no controle da dor em cães no pós-operatório. Ciência Veterinária UniFil, 1(2).

Spugnini, E. P., Dragonetti, E., Vincenzi, B. et al. (Ed) (2006). Pulse-mediated chemotherapy enhances local control and survival in a spontaneous canine model of primary mucosal melanoma. Melanoma Research. 16, 23-27.

Zeferino, A. R. G. (2019). Efeitos adversos associados à eletroquimioterapia em gatos com carcinoma das células escamosas: Estudo retrospectivo (Mestrado Integrado em Medicina Veterinária). Universidade Lusófona de Humanidades e Tecnologias, Lisboa, Portugal. 